\title{
Medium term results of lung transplantation for end stage pulmonary sarcoidosis
}

Stuart Walker, Ghada Mikhail, Nicholas Banner, John Partridge, Asghar Khaghani, Margaret Burke, Magdi Yacoub

\begin{abstract}
Background-Lung transplantation is an accepted therapeutic option for patients with end stage pulmonary sarcoidosis. However, the medium term outcome of transplantation in this patient group is unknown.

Methods-This study was performed to evaluate our experience with lung transplantation for end stage pulmonary sarcoidosis. Between July 1988 and July 1997 12 patients (nine men) underwent lung transplantation for sarcoidosis at our institution. Ten underwent single lung transplantation and two double lung transplantation.

Results-Survival at three and five years was $70 \%$ and $56 \%$, respectively. Three patients developed obliterative bronchiolitis at six, 18, and 45 months. One died at the time of retransplantation. Sarcoid granulomas have recurred in the donor organ in three patients. In one the development of granulomas has been associated with clinical deterioration, necessitating retransplantation. Mean (SD) forced expiratory volumes in one second at three and five years were 1.37 $(0.67) 1$ and $1.34(0.13) 1$, respectively.

Conclusions-Lung transplantation is a viable option for patients with end stage pulmonary sarcoidosis. The medium term results are comparable with patients undergoing lung transplantation for other indications. Despite histological recurrence of sarcoidosis, the risk of clinically important recurrence is low.

(Thorax 1998;53:281-284)
\end{abstract}

Keywords: lung transplantation; sarcoidosis; recurrence

Harefield Hospital,

Harefield, Middlesex

UB9 6JH, UK

$S$ Walker

G Mikhail

N Banner

J Partridge

A Khaghani

M Burke

M Yacoub

Correspondence to:

Dr S Walker, Department of

Cardiology, Harefield

Hospital, Harefield,

Middlesex UB9 6JH, UK.

Received 4 September 1997

Returned to authors
1 December 1997

Revised version received

5 January 1998

Accepted for publication

6 January 1998
Irreversible respiratory failure is an uncommon complication of sarcoidosis, occurring in less than $10 \%$ of those affected with this disorder. ${ }^{1}$ Whilst some patients with severe disease respond to intensive medical therapy with corticosteroids and other immunosuppressants, a small proportion of patients make no improvement. ${ }^{2}$ In this group lung transplantation is a potentially valuable form of therapy. Although transplantation for sarcoidosis has been shown to produce good early results, ${ }^{34}$ the medium and long term outcome has not been fully evaluated. This paper reports our experience over the last nine years with lung transplantation for respiratory failure caused by sarcoidosis.
Methods

PATIENTS

Between July 1988 and July 1997277 patients underwent lung transplantation for a variety of indications at Harefield Hospital. Of these, 12 patients $(4.3 \%)$, nine of whom were men, had an underlying diagnosis of sarcoidosis. Their mean age was 46.5 (9.6) years (range 33-60). Sarcoidosis was diagnosed using a combination of clinical, radiological (fig 1) and histological criteria (lung biopsy $(\mathrm{n}=11)$, Kveim test $(\mathrm{n}=$ 1)). ${ }^{5}$ Preoperatively all 12 patients were severely symptomatic. Seven patients suffered marked limitation of physical activity whilst five were unable to perform any physical activity without symptoms. Five were dependent on continuous oxygen therapy. One became ventilator dependent for the 38 days before transplantation, following complications after a spontaneous pneumothorax. Six were still receiving prednisolone therapy at the time of operation. The mean (SD) dose was 10.8 (4.8) $\mathrm{mg}$ /day (range 5-20 mg/day).

DONOR SELECTION AND SURGICAL TECHNIQUE Our donor selection criteria have been described previously. ${ }^{6}$ The mean donor age was 33.2 (12.2) years (range 17-44). Seven of the donors were women. Causes of death were intracranial haemorrhage ( $n=7)$, head injury $(n=3)$, and intracerebral abscess $(n=1)$. They had been ventilated for a mean of 2.33 (1.1) days (range 1-5). The mean total ischaemic time was 252 (61) minutes (range 157-350). The operative technique of single and double lung transplantation has been described previously. ${ }^{6}$ Ten patients underwent single lung transplantation and two underwent double lung transplantation. Of the patients

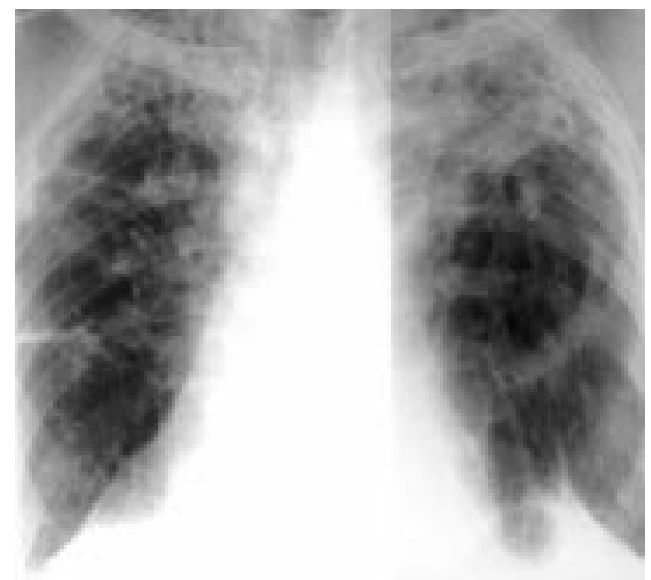

Figure 1 Chest radiograph showing chronic fibrotic pulmonary disease secondary to sarcoidosis prior to transplantation. 

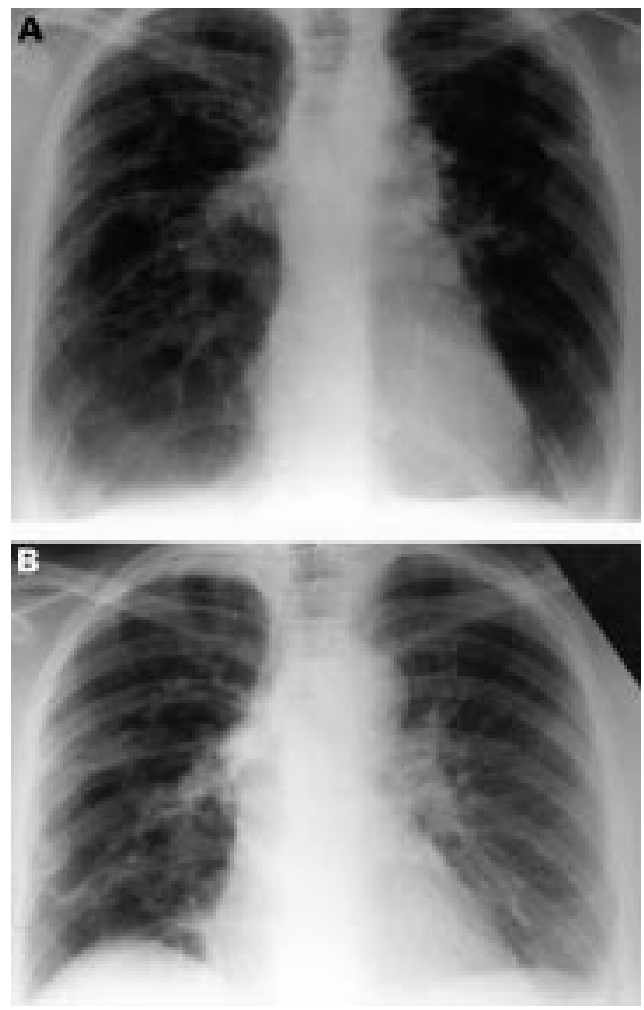

Figure 2 (A) Radiograph before transplantation showing an unusual degree of hyperinflation of both lungs. (B) Same patient as in $(A)$ one year after satisfactory left single lung transplantation. Note that the right lung is no longer hyperinflated.

undergoing double lung transplantation one had bilateral bronchiectatic changes whilst the other had bilateral bullous disease in association with sarcoidosis. The period of postoperative ventilation was 2.9 (3.4) days (range 12 hours to 11 days).

\section{IMMUNOSUPPRESSION}

Preoperatively each patient received oral cyclosporin at a dose of 3-6 mg/kg depending on renal function and oral azathioprine at a dose of $4 \mathrm{mg} / \mathrm{kg}$. One gram of intravenous methylprednisolone was given peroperatively. Subse-

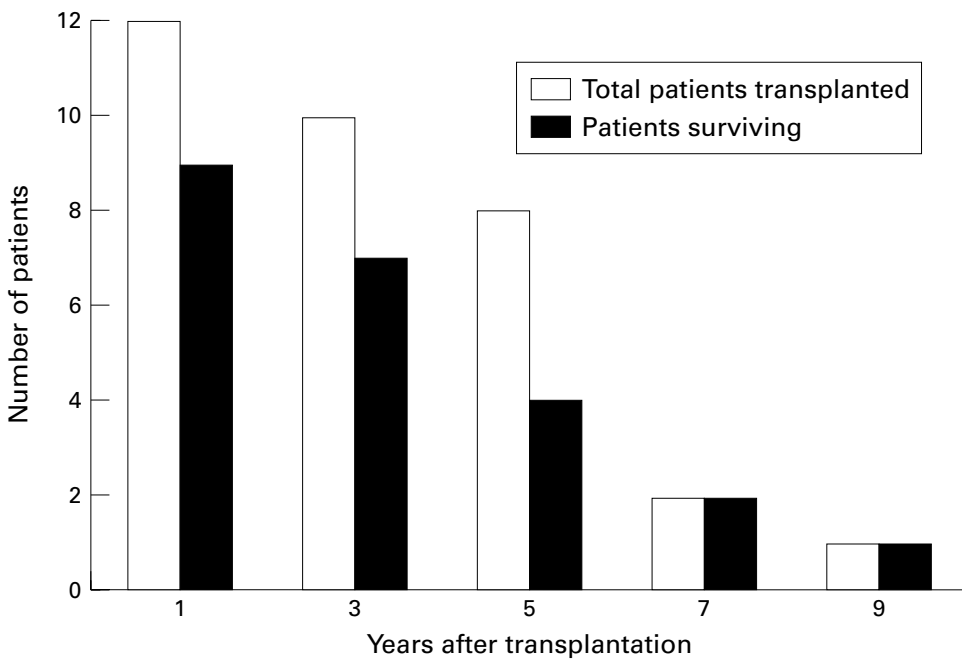

Figure 3 Medium term survival of patients undergoing lung transplantation for end stage pulmonary sarcoidosis. quently, cyclosporin was given twice daily to maintain a serum trough level of $450-550 \mathrm{ng} /$ $\mathrm{ml}$ for the first month. This target level was subsequently reduced over the next 11 months to a level of $200-250 \mathrm{ng} / \mathrm{ml}$. Azathioprine was continued at $2 \mathrm{mg} / \mathrm{kg} /$ day provided the white cell count was maintained above $4 \times 10^{9} / 1$. Oral steroids were given postoperatively in a reducing dose from $1 \mathrm{mg} / \mathrm{kg} /$ day to zero as clinically indicated. Prednisolone was continued in those with recurrent rejection or in those in whom full doses of cyclosporin and azathioprine could not be used because of drug side effects. Five patients required long term steroid therapy.

\section{POSTOPERATIVE CARE}

Bronchoscopy was performed 7-10 days postoperatively to examine the bronchial anastomosis, and subsequently when clinically indicated. Full pulmonary function tests were performed twice weekly during the first month, weekly until three months, and then two weekly until six months. Daily spirometric tests were performed at home by the patients using portable spirometers. At annual assessment pulmonary function tests, chest radiography (fig $2 \mathrm{~A}$ and $\mathrm{B}$ ), and exercise tolerance testing were performed. Acute rejection episodes were diagnosed using standard clinical, radiological, physiological, and histological criteria. ${ }^{7}$ Obliterative bronchiolitis syndrome was defined, using standard clinical criteria, as a late deterioration in lung function resulting in symptomatic deterioration in the absence of infection or acute rejection. In keeping with current guidelines, typical pathological changes on biopsy specimens were not required to make the diagnosis. ${ }^{8}$ Recurrence of sarcoid granulomas in the transplanted lung was defined as the appearance of non-caseating granulomas on a transbronchial biopsy specimen in the absence of mycobacterial, fungal, or pneumocystis infection.

\section{Results}

SURVIVAL AND OBLITERATIVE BRONCHIOLITIS SYNDROME

Three patients, all single lung transplant recipients, died in the first month. Two died peroperatively from acute donor organ malfunction. At post mortem examination sarcoidosis was confirmed as the underlying condition in both patients. The third died 11 days after surgery from respiratory failure secondary to acute donor organ malfunction and bilateral pneumothoraces. Post mortem examination was not performed. Of the remaining nine patients, eight are still alive at 11-105 months of follow up. In the first postoperative year the patients suffered an average of 3.1 (range 0-6) acute rejection episodes. Obliterative bronchiolitis syndrome developed in three cases at six, 18 , and 45 months. The syndrome grades were $1 \mathrm{~B}, 3 \mathrm{~A}$, and $1 \mathrm{~A}$ respectively. ${ }^{8}$ Of these three patients, one is awaiting retransplantation. One (a double lung transplant recipient) died at 39 months during a subsequent single lung transplantation. The third is clinically stable. The medium term survival is shown in fig 3 . 
Table 1 Mean (SD) preoperative and postoperative forced expiratory volume in one second (FEV ) and forced vital capacity (FVC)

\begin{tabular}{llll}
\hline & $\begin{array}{l}\text { No. of } \\
\text { patients }\end{array}$ & FEV $V_{1}(l)$ & FVC (l) \\
\hline $\begin{array}{l}\text { Preoperatively } \\
\text { Postoperatively }\end{array}$ & 7 & $0.85(0.34)$ & $1.77(0.66)$ \\
$\quad 3$ months & 7 & $1.77(0.32)$ & $2.50(0.52)$ \\
6 months & 7 & $1.75(0.44)$ & $2.55(0.52)$ \\
12 months & 6 & $2.10(0.75)$ & $2.95(0.79)$ \\
36 months & 5 & $1.37(0.67)$ & $2.15(0.90)$ \\
60 months & 2 & $1.34(0.13)$ & $2.09(0.32)$ \\
\hline
\end{tabular}

RESPIRATORY FUNCTION TESTS

Mean preoperative and postoperative pulmonary function test results are shown in table 1 .

RECURRENCE OF SARCOID GRANULOMAS IN THE TRANSPLANTED LUNG

No biopsies were performed to look for recurrent granulomas, but in the seven patients followed up for more than one year non-caseating granulomas were identified in three (all single lung transplants). These were identified in transbronchial biopsies undertaken for other indications. In all three patients infection with
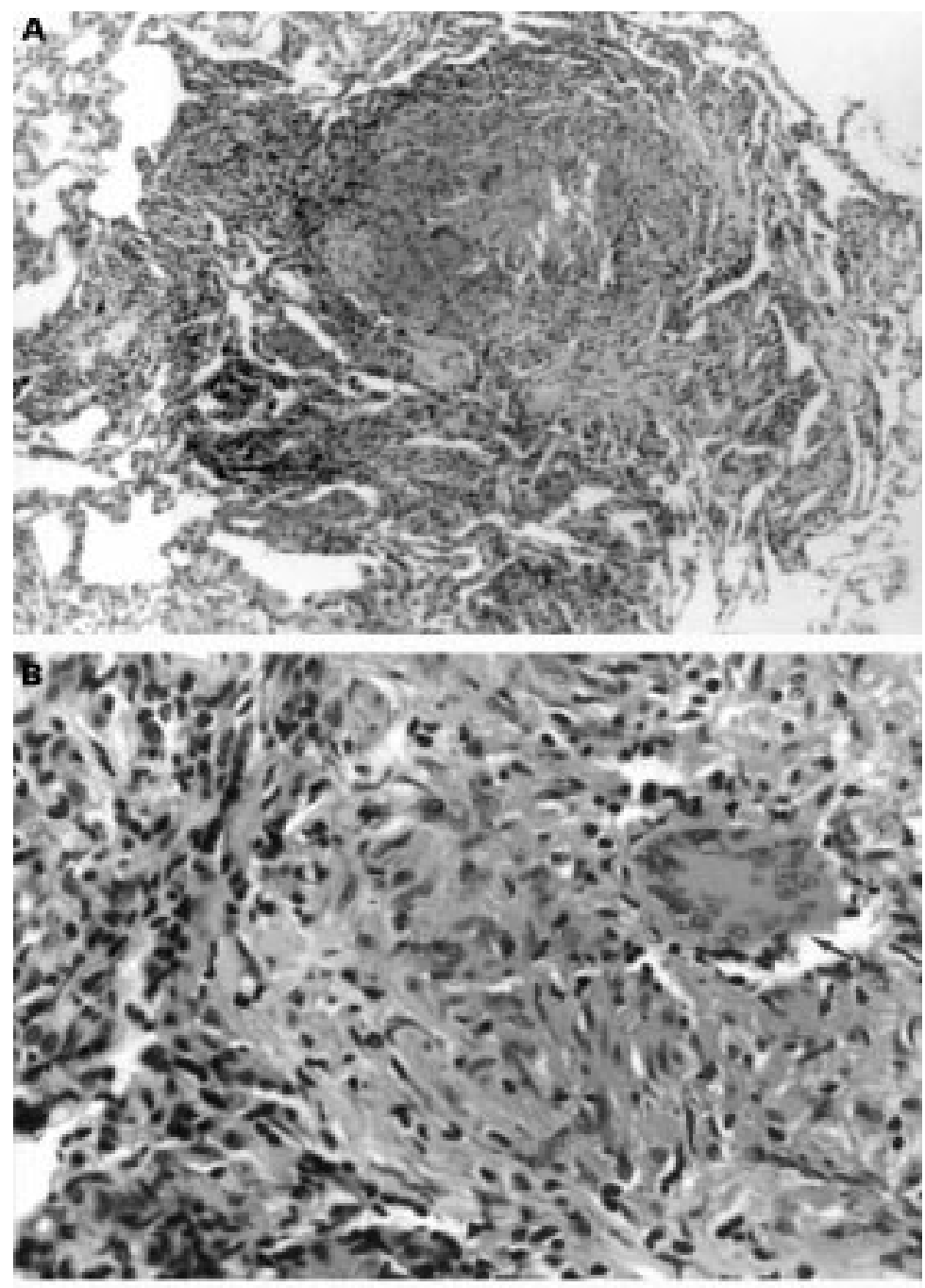

Figure 4 (A) A large interstitial non-caseating epithelioid granuloma with a diffuse interstitial lymphocytic infiltrate to the left. Stain: haematoxylin and eosin, magnification $\times 50$. (B) The edge of the granuloma showing a multinucleated giant cell (arrow) and many epithelioid macrophages. Stain: haematoxylin and eosin, magnification $\times 400$. mycobacteria, fungi, and Pneumocystis carinii were excluded by appropriate histological stains and microbiological techniques before a diagnosis of recurrent sarcoidosis was made. The granulomas were identified at five, six, and 56 months after transplantation.

In the first patient a biopsy was performed for a history of increasing dyspnoea and a decreasing forced expiratory volume in one second $\left(\mathrm{FEV}_{1}\right)$. A chest radiograph taken before the biopsy was unremarkable. CT scanning was not performed. The biopsy specimen was normal other than two interstitial noncaseating epithelioid granulomas, including multinucleated giant cells. The patient was treated with intravenous methylprednisolone. His symptoms resolved and no granulomas were identified on subsequent biopsies.

The second patient presented with increasing dyspnoea and cough associated with a fall in $\mathrm{FEV}_{1}$. His chest radiograph on admission was unremarkable. CT scanning showed no interstitial lung change or lymphadenopathy. Transbronchial biopsy specimens indicated active and organising pneumonitis and interstitial, perivascular, and peribronchiolar noncaseating epithelioid granulomas associated with a sparse lymphocytic infiltrate. His symptoms and $\mathrm{FEV}_{1}$ improved after three $1 \mathrm{~g}$ boluses of intravenous methylprednisolone followed by oral prednisolone in a reducing dosage. Unfortunately his symptoms returned after one month and a repeat biopsy demonstrated further interstitial non-caseating granulomas, this time with an adjacent diffuse interstitial lymphocytic infiltrate (fig 4). Despite conversion from cyclosporin to tacrolimus and treatment with intravenous and subsequent high dose oral steroids, his $\mathrm{FEV}_{1}$ remained at below $50 \%$ of his baseline value. $\mathrm{He}$ has recently undergone a second single lung transplantation for recurrent sarcoidosis.

The third patient underwent bronchoscopy for acute haemoptysis. Blood was seen to be originating from his native lung. A biopsy specimen taken from the donor lung at this time demonstrated bronchiolar submucosal non-caseating granulomas with multinucleated giant cells and very sparse small lymphocytes. No cause of the haemoptysis was identified at the time of bronchoscopy. Pseudomonas was grown in the bronchoalveolar lavage fluid taken from the native lung. Haemoptysis resolved after a course of antibiotic therapy. No granulomas were seen on subsequent biopsy specimens. CT scanning was not performed.

\section{EXERCISE TESTS}

Seven patients underwent exercise tolerance tests. Initially a cycle ergometer protocol was used, but this was changed to a treadmill protocol in 1991. Either the Bruce or modified Bruce protocols were used, depending on the patient's clinical condition. The tests were performed at three months, six months, and thereafter six monthly (table 2). Survivors achieved a reasonable level of rehabilitation with exercise capacities, determined by $\dot{\mathrm{V}}_{2} \mathrm{max}$, similar to those achieved after lung transplantation for other indications. ${ }^{9}$ These 
Table 2 Mean (SD) postoperative oxygen consumption on maximal exercise testing ( $\dot{\mathrm{V}}_{2}$ max)

\begin{tabular}{lll}
\hline & No. of patients & $\dot{V}_{2} \max (\mathrm{ml} / \mathrm{min} / \mathrm{kg})$ \\
\hline 3 months & 6 & $15.5(3.3)$ \\
6 months & 5 & $16.9(2.5)$ \\
12 months & 3 & $15.1(1.1)$ \\
18 months & 1 & 17.6 \\
24 months & 2 & $16.2(2.7)$ \\
36 months & 1 & 19.5 \\
48 months & 1 & 19.5 \\
60 months & 1 & 18.2 \\
\hline
\end{tabular}

exercise capacities have tended to be stable over the period of follow up.

\section{Discussion}

This study has documented the pattern of survival and clinical progress of a group of patients undergoing lung transplantation for sarcoidosis over a period of up to eight years. The worldwide overall actuarial survival in lung transplantation has recently been reported as approximately $72 \%$ at one year and $47 \%$ at four years, ${ }^{10}$ the most serious complication being the late development of obliterative bronchiolitis. ${ }^{11-13}$ We have demonstrated survival after transplantation similar to these results in our group of patients. However, when the international results are analysed further, patients with an underlying diagnosis of pulmonary fibrosis fare slightly less well than those with emphysema and alpha-1-antitrypsin deficiency. ${ }^{10}$ This may well reflect the fact that the underlying causes of pulmonary fibrosis, such as sarcoidosis or rheumatoid lung disease, are systemic disorders with an associated risk of recurrence after transplantation. We have not shown a worse outcome in our group despite this risk of recurrence.

Cases of histological recurrence of sarcoidosis have been reported in recipients of lung, ${ }^{41-19}$ heart, ${ }^{20}$ and kidney ${ }^{21}$ transplants. Our literature review has identified four cases of recurrent sarcoidosis associated with clinical deterioration and 17 without deterioration in 29 lung transplant recipients with sarcoidosis. Diagnosing clinical recurrence of sarcoidosis may be difficult because of the need to differentiate it from chronic rejection or because anti-rejection immunosuppression may be sufficient to prevent disease recurrence and progression. Our experience is of three histological recurrences in seven patients followed up for more than one year. In one patient the appearance of granulomas has been associated with an irreversible decline in lung function despite aggressive medical therapy, including increasing immunosuppression. In the other two patients the appearance of granulomas has been transitory and was not associated with serious clinical problems. It is concluded that, whilst histological recurrence is common after lung transplantation for sarcoidosis, this is usually not of clinical significance.

Two of our patients underwent double lung transplantation and the remainder underwent single lung transplantation. The number of patients in these groups is too small for meaningful comparisons between double and single lung transplantation. However, in view of the satisfactory results we have achieved with single lung transplantation for end stage sarcoidosis and the current paucity of donor organs, we usually recommend single lung transplantation for this condition.

In conclusion, the medium term results of lung transplantation for respiratory failure caused by sarcoidosis are similar to those obtained in other parenchymal lung diseases. Histological recurrence of sarcoidosis occurred in three cases but in only one was this associated with clinical deterioration. Patients with severe lung disease due to sarcoidosis should be considered for lung transplantation.

1 Hillerdal G, Nou E, Osterman K, et al. Sarcoidosis: epidemiology and prognosis. Am Rev Respir Dis 1984;130:29-32.

2 Weissler JC. Southwestern Internal Medicine Conference: Sarcoidosis: immunology and clinical management. Am $\mathcal{f}$ Med Sci 1994;307:233-45.

3 Scott J, Higgenbottom T. Transplantation of the lungs and heart for patients with severe pulmonary complications from sarcoidosis. Sarcoidosis 1990;7:9-11.

4 Johnson BA, Duncan SR, Ohori NP, et al. Recurrence of sarcoidosis in pulmonary allograft recipients. Am Rev Respir Dis 1993;148:1373-7.

5 DeRemee RA. Sarcoidosis: concise review for primary care physicians. Mayo Clin Proc 1995;70:177-81.

6 Yacoub MH, Banner NR. Recent developments in lung and heart-lung transplantation. In: Morris PJ, Tilney NE, eds. Transplantation reviews. Vol 3. Philadelphia: WB Saunders, 1989: 1-29.

7 Khaghani A, Banner NR, Ozdogan E, et al. Medium term results of combined heart and lung transplantation for emphysema. F Heart Lung Transplant 1991;10:15-21.

8 Cooper JD, Billingham M, Egan T, et al. A working formulation for the standardisation of nomenclature and for clinical staging of chronic dysfunction in lung allografts. $\mathcal{F}$ Heart Lung Transplant 1993;12:713-6.

9 Orens JB, Becker FS, Lynch JP 3rd, et al. Cardiopulmonary exercise testing following allogeneic lung transplantation for different underlying disease states. Chest 1995;107:1449.

10 Hosenpud JD, Bennett LE, Keck B, et al. The Registry of the International Society for Heart and Lung Transplantation: Fourteenth official report-1997. F Heart Lung Transplant 1997;16:691-712.

11 Burke CM, Theodore J, Baldwin JC, et al. Twenty eight cases of human heart-lung transplantation. Lancet 1986; 140:62-7.

12 McCarthy PM, Shumway NE. Improved survival after heart lung transplantation. $\mathcal{F}$ Thorac Cardiovasc Surg 1990;99:5460 .

13 Madden B, Radley-Smith R, Hodson M, et al. Medium term results of heart and lung transplantation. $\mathcal{F}$ Heart Lung Transplant 1992;11:S241-3.

14 Yeatman M, McNeil, Smith JA, et al. Lung transplantation in patients with systemic diseases: an eleven-year experience at Papworth Hospital. F Heart Lung Transplant 1996;15:144-9.

15 Martinez FJ, Orens JB, Deeb M, et al. Recurrence of sarcoidosis following bilateral allogenic lung transplantation. Chest 1994;106:1597-9.

16 Carre P, Rouquette I, Durand D, et al. Recurrence of sarcoidosis in a human lung allograft. Transplantation Proc 1995;27:1686.

17 Bjortutt O, Foerster A, Boe J, et al. Single lung transplantation as treatment for end stage pulmonary sarcoidosis: recurrence of sarcoidosis in two different lung allografts in one patient. F Heart Lung Transplant 1994;13:24-9.

18 Kazerooni EA, Cascade PN. Recurrent miliary sarcoidosis after lung transplantation. Radiology 1995;194:913.

19 Nunley DR, Keenan RJ, Dauber JH, et al. Lung transplantation for sarcoidosis at the University of Pittsburgh. $\mathcal{F}$ Heart Lung Transplant 1996;15:S42.

20 Oni AA, Hershberger RE, Norman DJ, et al. Recurrence of sarcoidosis in a cardiac allograft: control with augmented corticosteroids. F Heart Lung Transplant 1992;11:367-9.

21 Brown JH, Jos V, Newstead CG, et al. Sarcoid like granulomata in a renal transplant Nephrol Dialysis Transplant 1992; $7: 173$. 